\title{
A Conceptual Modeling Method of Simulation System Based on MCM
}

\author{
Bin $\mathrm{Liu}^{1, \mathrm{a}}$, Hongqiang $\mathrm{Gu}^{1, \mathrm{~b}}$ and Haiqing Wang ${ }^{1, \mathrm{c}}$ \\ ${ }^{1}$ Department of Equipment Command \& Management, Mechanical Engineering College, \\ Shijiazhuang 050003, China. \\ arobin8402@163.com, 'b shubainian@sina.com, 'wanghaiqing@163.com
}

Keywords: Meta conceptual model, Ontology, Unified Modeling Language (UML).

\begin{abstract}
Conceptual model is considered to be essential in simulation development activities. However, there are only a few research studies on how to develop conceptual models, the problems of model's low reusability and lacking management are presented in the conceptual model's development of simulation system. To solve the above problems, the concept of meta conceptual model (MCM) is presented for realizing the conceptual model's abstract in the higher arrangement. The ontology conception is introduced to design the MCM, and the MCM's architecture and modeling process are given too. By mapping the MCM to conceptual model (CM), the conceptual model's modeling process is realized. Finally, the method is applied in the conceptual model's modeling of equipment support simulation system, and the better validation is obtained.
\end{abstract}

\section{Introduction}

Conceptual modeling is known to be essential in simulation development to provide a better understanding of the system in early stages of development, and in this way to increase quality of requirements, enhance communication between users and developers and help in verification and validation of software products. Additionally, conceptual model acts as a basis to judge interoperability among distributed simulation systems [1]. However, the problems of model's low reusability and lacking management have existed in the conceptual modeling process, which may influence the conceptual model's efficiency and simulation system's reliability immediately [2].

In order to resolve the above problems, the concept of Meta Conceptual Model (MCM) is given in this paper, and the ontology idea is used for the MCM's design. By realizing the CM's abstractions in the higher arrangement, and describing the CM's syntax and semantic, the CM's reusability is improved.

\section{Related Concepts}

Meta Conceptual Model. "Meta" means overtaking or basic, which often be used with other nouns such as language, model and data. The concepts of meta language, meta model and meta data are formed. When developing the conceptual models of some specific domains, the "meta" may be used before CM, and the concept of meta conceptual model (MCM) is presented. In brief, MCM is the model of CM and has the higher abstract degree. By using the MOF (Meta Object Facility) for reference [3], the four levels MCM architecture is presented, which is shown in Table 1.

Table 1 MCM's Architecture

\begin{tabular}{cc}
\hline Levels & Description \\
\hline $\begin{array}{c}\text { Meta Meta CM (M3) } \\
\text { Meta CM (M2) }\end{array}$ & $\begin{array}{c}\text { Define the language for describing MCM } \\
\text { A instance of M3, Define the language for } \\
\text { describing CM }\end{array}$ \\
CM (M1) & $\begin{array}{c}\text { A instance of M2, Define the language for } \\
\text { describing real word }\end{array}$ \\
Real Word (M0) & A instance of M1, describe real word \\
\hline
\end{tabular}


Ontology. The concept of Ontology raise in philosophy field, which was used in the field of computer and artificial intelligence in the later 20 century [4]. There are many different of knowledge about the definition of ontology, but the basic knowledge was that ontology is the distinct standard explanation about a level of the knowledge of theme (field). The goal of developing ontology is getting, describing and expressing related field knowledge, offering the common understanding for this field knowledge, and determining the vocabulary in this field that approved together.

Ontology description language (ODL) is used to express and describe Ontology, such as Ontolingua, Cycl, Loom, KL-ONE and OWL. These ODLs have rich expressing and inference ability, but they are hard to understand and be grasped by ordinary users for having adopted plenty of logic symbol and complex grammar. Therefore these methods still have greater restriction on application. UML (Unified Modeling Language) is a representative language and method for oriented object modeling. By using the UML as a bridge for modeling, the ontology description frame may be built. Especially the class chart has offered rich graphic symbol to define the relation between concept as well as the concept in field ontology, which makes carrying out body using object oriented way build mould to become possibility [5].

Ontology-based MCM. As known from the above content, the ontology is a formal, explicit specification of a shared conceptualization. Conceptualization refers to an abstract model of some phenomenon in the world by having identified the relevant concepts of that phenomenon. Therefore, the MCM established by using ontology models may describe the domain concept and relation between concepts, be an effective way to resolve the conceptual modeling's reusability problem. Based on above analysis, the ontology thinks may be led into MCM's building, and the OMCM (Ontology-based Meta Conceptual Model) is formed.

The traditional object-oriented modeling methods have been mainly understand the meta modeling framework and instantiation relation with linguistics meaning, lacking enough semantic description ability and relation between meta-meta model and real word. By using OMCM method, the semantic oriented MCM may be built, which may realize the MCM's usability. At the same time, object oriented modeling method lacks effective model validation mechanism, and be hard to ensure the availability and consistency between various of terminologies (concepts). The OMCM may realize these by using ontology reasoning mechanism.

\section{Modeling Process}

As shown in Fig. 1, the MCM modeling process includes two steps: constructing ontology and constructing MCM.

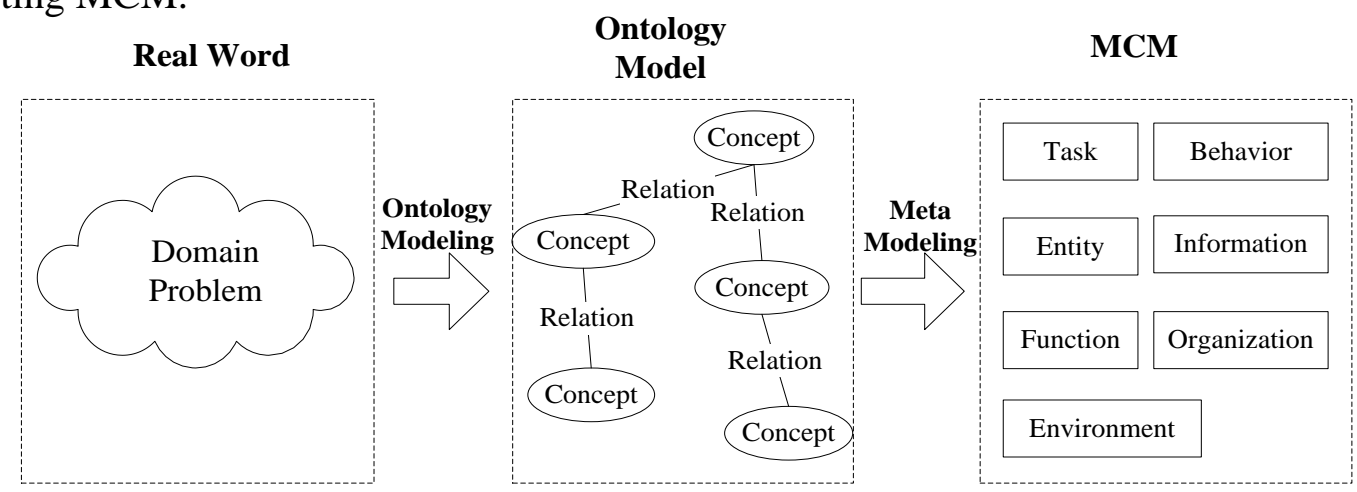

Fig. 1 MCM Modeling Process

In order to develop the ontology, the users must do some transformations to obtain the ontology models. At present, the ontology development pattern include: USchold\&King method [6], Gruninger and Fox method [7], METHONTOLOGY method [8] and so on. By analyzing these methods, ontology development method include four steps: domain knowledge acquisition, domain knowledge representation, domain knowledge modeling, and domain ontology accreditation.

Based on the ontology above, the MCM development method may include three steps: MCM factor's acquisition, ontology-MCM mapping and MCM description. 


\section{Step 1 MCM factor's acquisition}

MCM factor is the meta concept which may reflect the domain's essence. In this paper, the multi-views methodology is used to collect the corresponding meta factor. One view means the domain model's space, which may describe the domain behavior's different sides. According to the theory of system science, the domain system may be divided into six views: component view, construction view, process view, function view, information view and environment view. Additionally, The entity meta concept, organization meta concept, process meta concept, behavior meta concept, function meta concept, information meta concept, and environment meta concept are acquisitioned.

Step2 Ontology-MCM mapping

After ascertaining the domain's meta concepts, the modeler should do some descriptions. In this paper, the ontology-MCM mapping method is given to describe the meta concepts. The ontology-MCM mapping way may be represented by following process: the basic ontology knowledge such as concept, attribution and relation should be defined firstly, then the knowledge's relation should be described. For example, the behavior meta concept is related to role, behavior, information concept, the role and behavior is Participate-In relation, the behavior and information is Work-Out relation.

Step 3 MCM description

According to Step 1, the domain system may be abstracted to seven basic factors, these factors are related to corresponding meta concept model (MCM), which are entity MCM, behavior MCM, task MCM, function MCM, information MCM, organization MCM and environment MCM. In this paper, the Unified Modeling Language (UML) is used to describe these MCM.

\section{The Mapping Between MCM and CM}

Mapping Process and Mapping Rules. The relation between MCM and CM is like the relation between Class and Object in Software Engineering. MCM is the essential of the CM, and the CM is the MCM's instantiation. In order to realize the transformation between MCM and CM, the mapping relation should be set up. A three element group is given, which may be described by the following formula: $\mathrm{M}=<\mathrm{SM}, \mathrm{TM}, \mathrm{R}>$. In this formula, $\mathrm{SM}$ is the source model-MCM, TM is the target model-CM, $\mathrm{R}$ is the mapping rules between MCM and CM. In order to represent the relation intuitively, the mapping process may be described by the Fig. 2.

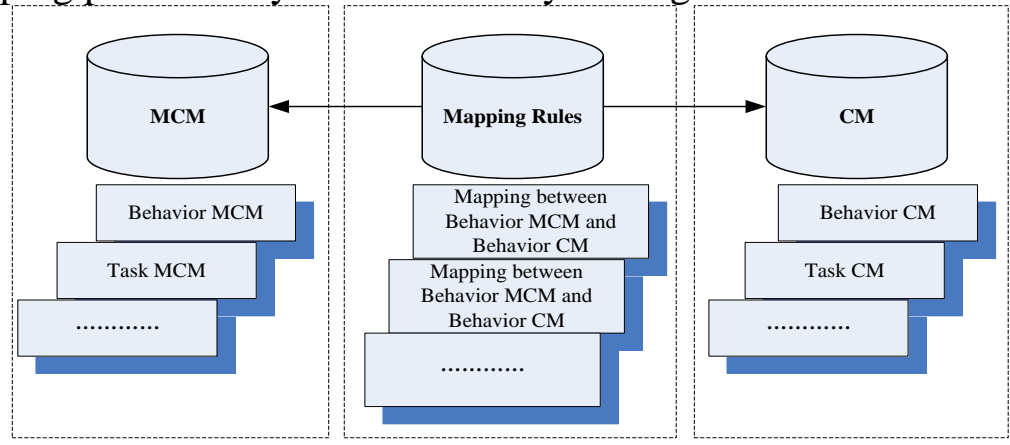

Fig. 2 The Mapping Process Between MCM and CM

Mapping rules is a set of methods and rules, which may be used to modified one model and get another model. In this paper, the mapping rules include three kinds of mappings: concept mapping, property mapping and relation mapping. Concept mapping represents the concepts' mapping transformation between MCM and CM, just as the Behavior MCM may be transformed to many kinds of CM. Property mapping is the transformation between MCM and CM's property. Relation mapping represents the relation's mapping between MCM and CM.

The Mapping Between MCM and UML. In this paper, the UML Class Diagram is used to construct MCM, and other UML Diagrams are used to construct CM. The UML model language include five kinds of diagrams: use case, static diagram, behavior diagram, interactive diagram and implementation diagram. In Table 2, the relations between CM and UML are shown. 
Table 2 The Relations Between CM and UML

\begin{tabular}{cc}
\hline CM & UML Model \\
\hline Function CM & Use case, Class diagram \\
Task CM & Use case, Class diagram \\
Entity CM & Class diagram \\
Organization CM & Class diagram \\
Behavior CM & Behavior diagram \\
Information CM & Class diagram, Sequence diagram \\
\hline Environment CM & Class diagram \\
\hline
\end{tabular}

\section{A Case Study}

The modeling method based on MCM may be used in many kinds of system's modeling. In this section, we take the equipment support system for example. Firstly, the ontology models of equipment support system will be developed. Secondly, the MCM of this system will be constructed. Finally, the CM of this system will be realized by the MCM's instantiation.

The Ontology of Equipment Support System. From the angle of knowledge engineering, the ontology of equipment support system is the conceptualization of the domain knowledge of equipment support system. According to the ontology modeling method, the concepts and relations of equipment support system are analyzed in this paper.

In order to develop ontology of equipment support system, the related concepts are represented by Class. The main concepts are included in the following concepts: Environment, Organization, Entity, Rule, Process, Function, Event, Resource, Information, Task, Behavior, Goal, Character. Every concepts include the related properties, which are represented by Class diagram. Because of the restriction of paper's length, we will not describe every concept in detail in this paper.

When describing the meta relation of equipment support system, except using the relations in UML (Association, Aggregation, Generalization, Dependency), we have constructed other relations according to the domain's characteristic, such as Trigger, Belong-To, Aim-To, Perform, Participate-In, Abide, Constrain, Own, Respond, Play, Work-Out, Change, Affect, Present-In, Decide.

The Equipment Support Simulation MCM. The equipment support simulation MCMs include the following seven parts: Entity MCM, Behavior MCM, Organization MCM, Information MCM, Function MCM, Task MCM, Environment MCM. The relations between them may be shown in Fig. 3.

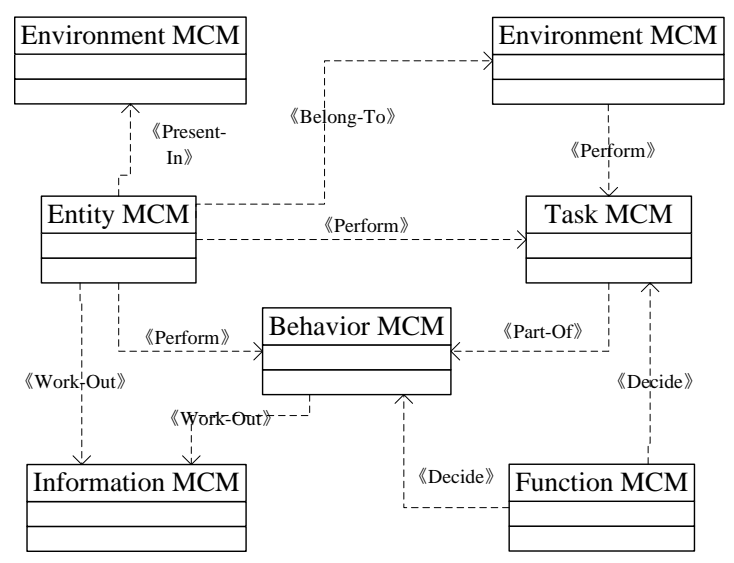

Fig. 3 Equipment Support Simulation MCM Description

In this paper, for describing these MCM, we take the Behavior MCM for example. The entity may perform some behaviors, which means it has the corresponding functions. Some kinds of behaviors are not usually performed isolatedly, instead of being subject to certain tasks. When the behaviors are performed, they are usually constrained by some rules and work out some information. Therefore, the 
concepts related with behavior include the following concepts: Function, Task, Rule, Event and Information, which may be shown in Fig. 4.

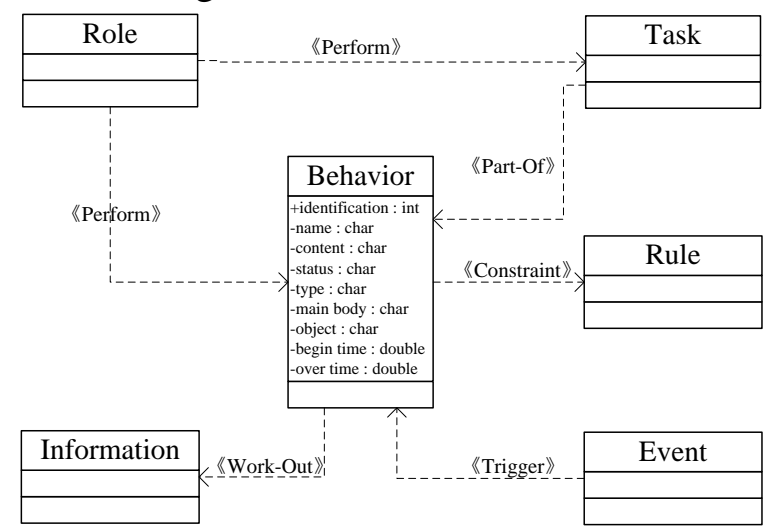

Fig. 4 Behavior MCM Description

Equipment Support Simulation CM. After developing the MCM of equipment support simulation, the mapping from MCM to CM should be carried out. the Move Behavior model is shown as an example to describe the conceptual modeling process.

In the UML method, UML activity diagram is one of the tools for system's dynamic modeling, whose main modeling idea root in Event Chart, SDL state modeling technique and Petri-net technique. The following factors are included: Initial Node, Final Node, Activity, Action, Swim lane, Object Node, Note, Control Flow, Object Flow, Fork/Join, Branch/Merge.

In order to carry out the mapping from Behavior MCM to Behavior CM, we should let the factors in them connected. As shown in Figure 4, the factors of Behavior MCM include: Behavior, Role, Task, Rule, Event, Information. The Behavior is related with activity in activity diagram. The Role is related with Swim lane in activity diagram, each part divided by swim lane represent different role. The Task may be regarded as a complex behavior, which may be represented by activity in activity diagram. The Rule is regarded as a criterion and restraint, may be described by Control Flow, Object Flow, Fork/Join, and Branch/Merge. Event is the encouragement for behavior's execution, may be represented by the event name in activity diagram. The Information represent the activity's interacts, which may be shown by the note in activity diagram. In this paper, the Move Behavior model is shown in Fig. 5.

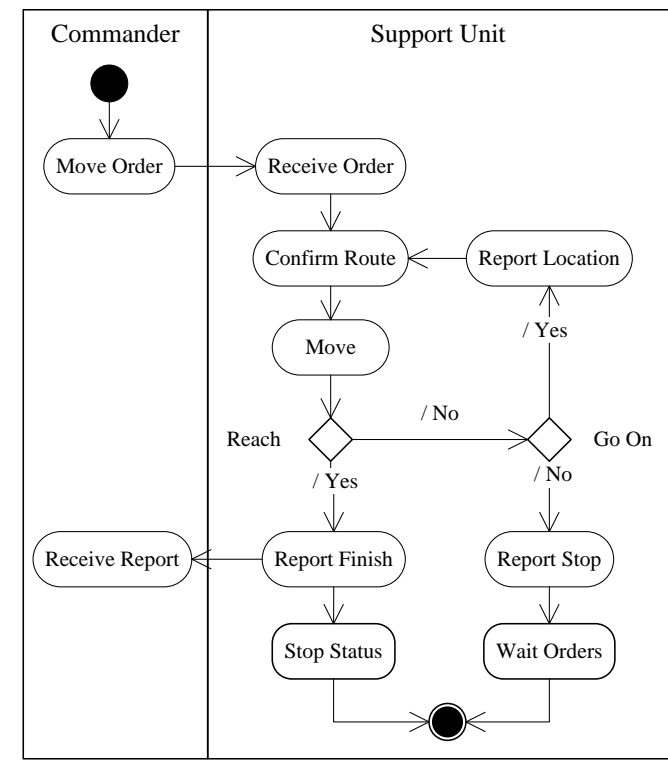

Fig. 5 Equipment Support Unit Move Process Model

Firstly, the limited communication devices are usually used by the fighting units, which results in the communication ability's lack and network invulnerability's bad.

Secondly, there are specific relationships between up and down in the equipment support information network, which will result in the network's invulnerability bad under selective attack. 
Thirdly, the network node can't connect easily because of the strict operation flow, and the connection skipped to the higher level is controlled strictly, which will result in the sparse network connection and generic invulnerability under random attack.

\section{Summary}

In order to resolve the problem in CM's development, the ontology idea and meta modeling method are brought in, and the concept of MCM based on ontology is proposed. This method has shown the better reusability. By mapping the MCM to CM, the CM's modeling is realized. This method has been used in the equipment support simulation system, the equipment support simulation CMs are constructed, and the method's feasibility and availability are confirmed.

\section{References}

[1] A. Tolk, J. A. Muguira, “The Levels of Conceptual Interoperability Model”, Proceedings of SISO Fall Simulation Interoperability Workshop, Orlando, FL, September 14-19, 2003.

[2] O. Balci, J. D. Arthur, R. E. Nance, "ACCOMPLISHING REUSE WITH A SIMULATION CONCEPTUAL MODEL", Proceedings of the 2008 Winter Simulation Conference, Miami, FL, 959-965, 2008.

[3] L. Heaton. OMG Meta Object Facility (MOF) 1.4 Specification. http://www.omg.org/mda/specs.htm.

[4] N. Guarino, P.Giaretta, "Ontologies and Knowledge Bases: Towards a Terminological Clarification”, MARS I. Towards very Large Knowledge Bases-Knowledge Building and Knowledge Sharing. N.J.: IOS Press, 25-32, 1995.

[5] J. Evermann, “A UML and OWL description of Bunge’s upper-level ontology model”, Software and Systems Modeling, vol. 8, No. 2, pp. 235-249, 2009.

[6]M. Uschold, M. King, “Towards a Methodology for Building Ontologies ”, Workshop on Basic Ontological Issues in Knowledge Sharing, IJCAI-95, 1995.

[7] M. Gruninger, M.Fox, "Methodology for the Design and Evaluation of Ontologies”, IJCAI Workshop on Basic Ontological Issues in Knowledge Sharing, Montreal, Quebec, Canada, 1995.

[8] M. Fernandez-Lopez, A. Gomez-Perez, A. Pazos-Sierra and J. Pazos-Sierra, "Building a Chemical Ontology Using Methontology and the Ontology Design Environment”, IEEE Intelligent Systems and their applications January/February: 37-46. 